\title{
Surface atomic structure determination of three-dimensional yttrium silicide epitaxially grown on $\operatorname{Si}(111)$
}

\author{
C. Rogero, P. L. de Andres, and J. A. Martín-Gago \\ Instituto Ciencia de Materiales de Madrid-CSIC, 28049-Cantoblanco, Spain
}

(Received 14 June 2004; published 11 April 2005)

\begin{abstract}
The surface atomic structure of thin layers of three-dimensional yttrium silicide epitaxially grown on $\mathrm{Si}(111)$ $7 \times 7$ has been investigated by means of dynamical low-energy electron diffraction analysis. We determine the interlayer distances as well as the lateral and/or vertical relaxations of the atoms in the superficial planes. The epitaxial silicide consists of stacked hexagonal rare-earth planes and graphitelike Si planes with an ordered arrangement of Si vacancies. The ordered net of Si vacancies in the inner planes is responsible for the lateral relaxations of the surrounding $\mathrm{Si}$ atoms. The topmost layer does not present a graphitelike structure, forming a buckled Si layer with no vacancies. One of the three Si atoms in the lower plane of this bilayer is closer to the yttrium layer due to the presence of the vacancy in the last Si plane just below. This produces vertical relaxation in the termination layer.
\end{abstract}

DOI: 10.1103/PhysRevB.71.165306

PACS number(s): 61.14.Hg, 82.45.Mp, 68.35.-p, 68.55.-a

\section{INTRODUCTION}

Heavy rare-earth (RE) silicides epitaxially grown on $n$-type $\mathrm{Si}(111)$ can be used in electronic devices (e.g., infrared detectors, ohmic contacts or rectifying contacts) because of their unusual low values for the Schottky barrier height, ${ }^{1-5}$ and very small lattice mismatch at the interface. ${ }^{6-9}$ Moreover, the abruptness of the silicide/vacuum interface makes them good candidates to grow new layers on top, offering new perspectives for integrated silicon technology. ${ }^{10}$ However, despite their technological relevance an important issue remains open: which is the atomic structure of these silicide surfaces? In this paper we perform a detailed surface structural study for the yttrium silicide by means of dynamical low-energy electron diffraction (LEED).

For thin RE silicides epitaxially grown on $\mathrm{Si}(111)$ several surface reconstructions have been reported for different coverages. If less than 1 monolayer (ML) is deposited, different reconstructions are visible, $(2 \sqrt{3} \times 2 \sqrt{3}) R 30^{\circ}$ or $(5 \times 2) .{ }^{11}$ For RE coverage of around $1 \mathrm{ML}$, these silicides present a two-dimensional (2D) metallic structure, exhibiting a $p(1 \times 1)$ periodicity. ${ }^{12}$ At higher coverage, a $(\sqrt{3} \times \sqrt{3}) R 30^{\circ}$ LEED pattern appears, and a three-dimensional (3D) metallic silicide is formed. ${ }^{13-19}$

The atomic structure of the inner silicide planes of these 3D metallic RE silicides grown on $\mathrm{Si}(111)$ is sketched in Fig. 1 (we will refer to that as the bulk structure in the present work). It consists of a hexagonal structure derived from the $\mathrm{AlB}_{2}$-type geometry: graphitelike $\mathrm{Si}$ planes intercalated with $\mathrm{RE}$ planes. The Si planes contain vacancies, forming an ordered $(\sqrt{3} \times \sqrt{3}) R 30^{\circ}$ network that leads to a $\operatorname{RESi}_{17}$ stoichiometry (in Fig. $1 \mathrm{Si}$ vacancies are marked as stars). ${ }^{14,15}$ Vacancies play an important role in the atomic and electronic structure of these silicides, ${ }^{20}$ they release the compressive strain caused by the absence of buckling in the Si planes. ${ }^{21}$ If $c=4.14 \AA$ is the distance between two Si planes (or between two RE planes) in the direction perpendicular to the surface, the crystal periodicity is $2 c$ (upper panel of Fig. 1). Therefore, the geometry varies for two contiguous silicon planes due to the position of the $\mathrm{Si}$ vacancies. The next Si plane just below or just above of a referenced one has the same $(\sqrt{3} \times \sqrt{3}) R 30^{\circ}$ superstructure formed by the vacancies, but it is rotated around the surface normal. Ab initio theoretical calculations ${ }^{22}$ have demonstrated that it is possible to obtain similar total-energy values using different periodicities in the $z$ directions. Lattice parameters of $c, 2 c$ and even $3 c$ have been found compatible with the calculations. ${ }^{22}$ Similarly, a discrepancy exists in the value of the rotation of the vacancies around the surface normal. Although the most accepted model includes a rotation angle of $120^{\circ},{ }^{15,23}$ other angular values, like $60^{\circ}$, or $0^{\circ}$, have been published too. ${ }^{21}$

In the last years, theoretical $22,24,25$ and experimental studies ${ }^{16,21,26-29}$ have been carried out in order to determine the surface atomic positions in $\mathrm{RESi}_{1.7}$ epitaxially grown on
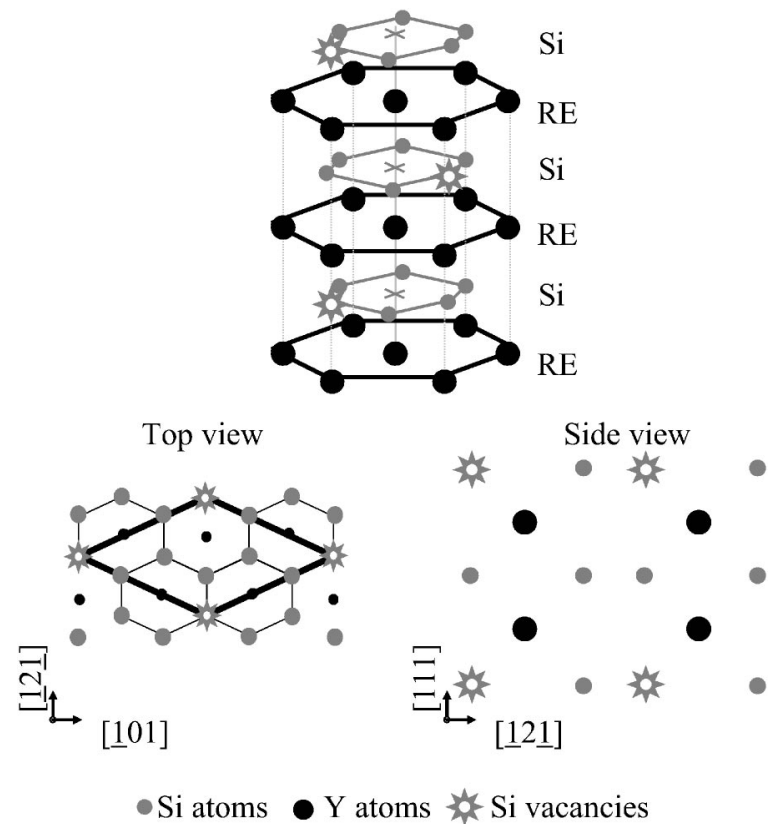

FIG. 1. Schematic representation of the bulk atomic structure of $\mathrm{YSi}_{1.7}$. The $\mathrm{Si}$ vacancies form a $(\sqrt{3} \times \sqrt{3}) R 30^{\circ}$ superstructure. 
(a) Roge et al. Model: $\mathrm{Si}_{\text {up }}$ over vac.

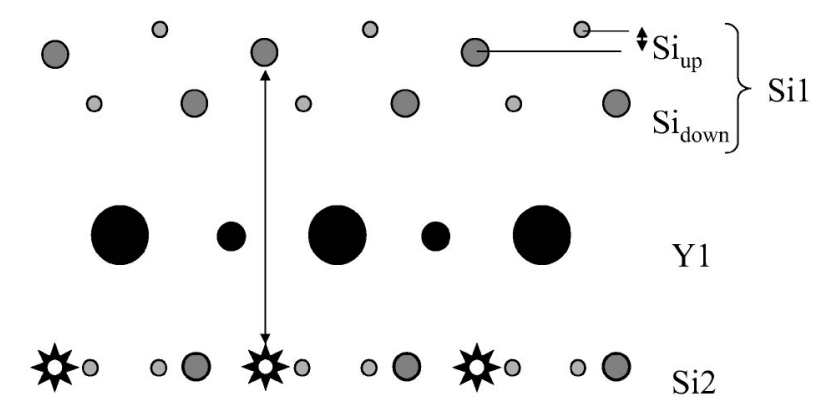

(b) Martin-Gago et al. Model: $\mathrm{Si}_{\mathrm{down}}$ over vac.

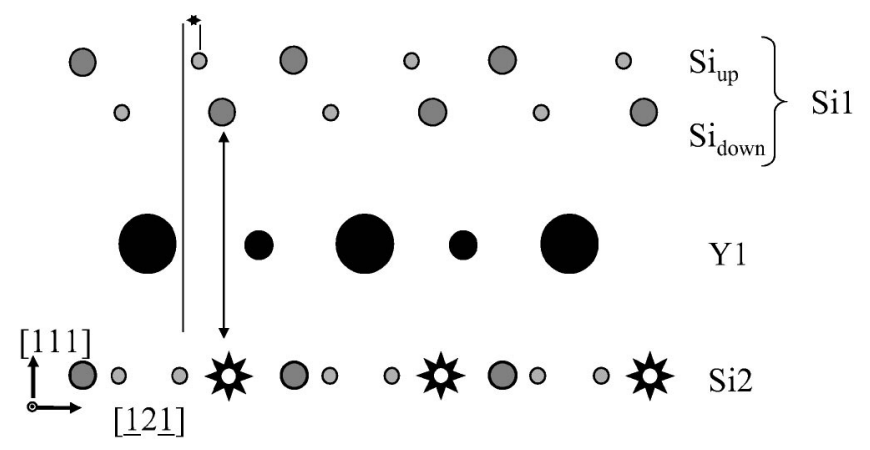

FIG. 2. (a) Surface atomic model proposed by Roge et al. (upper panel) (Refs. 31 and 32) in which the vacancies are located below the $\mathrm{Si}_{\text {up }}$ atoms, and (b) surface atomic model proposed by MartínGago et al. (lower panel) (Refs. 33 and 34) in which the vacancies are below the $\mathrm{Si}_{\text {down }}$.

$\mathrm{Si}(111)$. However, the atomic structure of the silicide surface has not been properly established until now. The presence of a Si bilayer termination was early determined by x-ray photoelectron diffraction (XPD) ${ }^{15,30}$ However, there is a discrepancy about the positions of the atoms in this bilayer and the influence of the vacancies below. Two different models, based on the atomic resolution scanning tunneling microscopy (STM) images of the surface of $\mathrm{ErSi}_{1.7}$ epitaxially grown on $\mathrm{Si}(111)$, were presented. The first geometry was proposed by Roge et al. ${ }^{31,32}$ The authors observed in their STM images that one of the three topmost $\mathrm{Si}$ atoms $\left(\mathrm{Si}_{\text {up }}\right)$ of the buckled layer appears to be lower than the other two. This observation led them to propose a geometry where one $\mathrm{Si}_{\text {up }}$ atom is sitting on top of the vacancy and, in consequence, inward relaxed with respect to the other two [this model is schematically shown in Fig. 2(a)]. The second model, proposed by Martín-Gago et al. (Refs. 33 and 34) and schematically shown in Fig. 2(b), is based on the observation of certain association of the three $\mathrm{Si}_{\text {up }}$ atoms forming triangular shaped atomic resolution STM images. The authors interpreted these groupings as a lateral relaxation of the topmost $\mathrm{Si}$ atoms. Instead of moving vertically, the three atoms would displace, by the same amount, towards the $\mathrm{Si}_{\text {down }}$ atom situated on top of the vacancy in the layer below. Both models were proposed on the basis of STM image analysis. Therefore, the determination of the position of the vacancies with respect to the $\mathrm{Si}$ atoms in the bilayer cannot be obtained. To elucidate this point, Magaud et al. ${ }^{22}$ carried out an $a b$ initio study concluding that vacancies were located under $\mathrm{Si}_{\text {down }}$, inducing a certain amount of lateral and vertical relaxations of the $\mathrm{Si}_{\text {up }}$ atoms.

In this paper we study the atomic configuration of the last silicide planes analyzing LEED-I(V) curves obtained for the surface of $\mathrm{YSi}_{1.7}$ epitaxially grown on $\mathrm{Si}(111)$. To find the surface geometry we have searched for a global minimum of the Pendry correlation $R$-factor. ${ }^{35}$ This standard procedure allows us to determine the values for structural and nonstructural parameters, finding accurate values of the atomic positions in the last surface planes.

\section{EXPERIMENTAL AND THEORETICAL DETAILS}

Experiments were performed at room temperature (RT), in an ultrahigh vacuum (UHV) system. The chamber is equipped with LEED optics, CMA for Auger electron spectroscopy (AES), and an STM working at RT. The combination of these techniques allows us to study the composition of the epitaxial layers, their surface morphology, ${ }^{36}$ and the atomic structure on the grown films. The base pressure was in the low $10^{-10} \mathrm{mbar}$, raising to $2 \times 10^{-9} \mathrm{mbar}$ during the $\mathrm{Y}$ evaporation.

The n-type $\mathrm{Si}(111)$ wafers used as substrate were cleaned in situ by standard methods (resistive heating up to $1200{ }^{\circ} \mathrm{C}$ followed by slow cooling), resulting in a sharp $7 \times 7$ LEED pattern. To form the 3D silicide, solid-phase epitaxy was used. ${ }^{36}$ The $\mathrm{Y}$ atoms were evaporated by electron bombardment and deposited at RT on the $\operatorname{Si}(111) 7 \times 7$ surface (the evaporation rate was monitored with a quartz balance). Samples were then annealed at $420^{\circ} \mathrm{C}$ for 10 minutes resulting in a silicide, characterized by a sharp $(\sqrt{3} \times \sqrt{3}) R 30^{\circ}$ LEED pattern. Preparation conditions were previously optimized, in order to obtain surfaces with large and flat terraces. ${ }^{36}$

A computer controlled 8 bits CCD camera has been used to collect the LEED IV curves. ${ }^{12,37}$ The spectra were recorded at room temperature and at normal incidence in an energy range from 50 to $450 \mathrm{eV}$, in $0.5 \mathrm{eV}$ steps. LEED-IV measurements have been taken on films with coverage around $10 \mathrm{ML}$, to make sure that the Si substrate does not contribute to the LEED-IV spectra. Using thick layers, we lose access to the Si-silicide interface, but the information on the silicide surface structure is of better quality. From this point of view, the so-called $(\sqrt{3} \times \sqrt{3}) R 30^{\circ}$ superstructure of the $3 \mathrm{D} \mathrm{RESi}_{1.7}$ referred to the ideal $\mathrm{Si}(111)$ substrate is, in fact, a $1 \times 1$ silicide LEED pattern. Therefore, although the usual notation identifies the silicide structure as a $(\sqrt{3} \times \sqrt{3}) R 30^{\circ}$, for the calculation we simulate the real structure, which is a nonreconstructed $1 \times 1$ with a base of eight atoms per unit cell, five $\mathrm{Si}$ atoms and three $\mathrm{Y}$ atoms. That implies the use of the lattice parameter of the superstructure, $a_{\mathrm{RESi}}=6.65 \AA, \sqrt{3}$ bigger than the lattice parameter of the $\mathrm{Si}$ substrate $\left(a_{\mathrm{Si}}=3.84 \AA\right)$, and the redefinition of the beam families. Using the conventional notation we have measured five families of diffracted beams: $(1 / 3,1 / 3),(2 / 3,2 / 3)$, $(1,0),(1,1)$, and $(2,0)$. These correspond to the following beams in the $1 \times 1$ structure: $(1,0),(2,0),(1,1),(3,0)$, and $(2,2)$, respectively. In this paper we use the former notation, 
(a) Side view

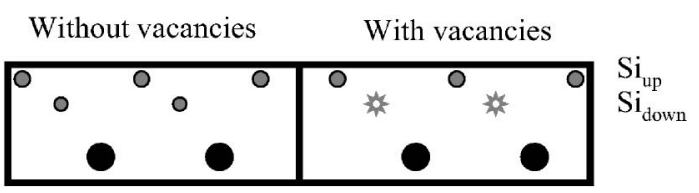

(b) Side view

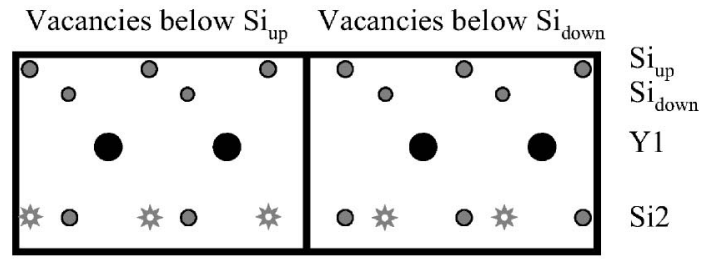

(c)

$0^{\circ}$ Rotated

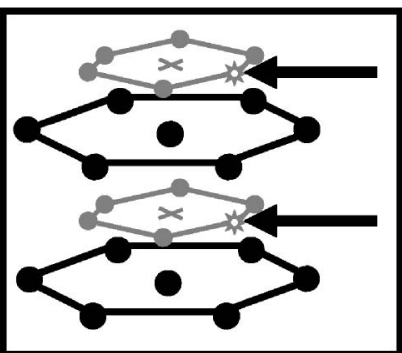

$60^{\circ}$ Rotated

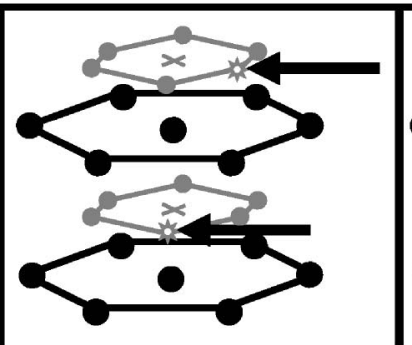

$120^{\circ}$ Rotated

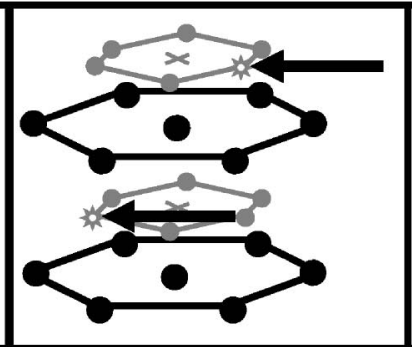

$180^{\circ}$ Rotated

FIG. 3. Schematic representation of the different possibilities we have tried for the structural determination of the $\mathrm{YSi}_{1.7}(\sqrt{3} \times \sqrt{3}) R 30^{\circ}$. We have studied (a) the presence or not of vacancies in the topmost buckled Si layer, (b) the staking of the vacancies with the $\mathrm{Si}_{\text {up }}$ or with the $\mathrm{Si}_{\text {down }}$ atoms, and (c) whether the vacancies are piled up or rotated $60^{\circ}, 120^{\circ}$, and $180^{\circ}$ in consecutive planes.

which is the common one used in the literature. The background was subtracted and equivalent spots were averaged to minimize noise in the curves. The size of the experimental database determines a statistical variance of $\mathrm{RRP}=0.02$ for our best fit structure. We notice that the $(\sqrt{3} \times \sqrt{3}) R 30^{\circ}$ superstructure has threefold rotational symmetry. However, the experimental LEED pattern of this silicide presents a sixfold rotational symmetry. This is related to the presence of different surface domains: As we will discuss in the next section, the existence of different domains is an extra difficulty for the accurate determination of the stacking of Si vacancies.

We have used the CLEED package ${ }^{38}$ for the calculation of dynamical LEED I(V) curves. Layer-doubling ${ }^{35}$ was used, switching to a real space composite layer method ${ }^{39}$ whenever the distance between layers is too short. The Pendry $R$-factor ${ }^{40}$ was selected to quantify the agreement between the theoretical and the experimental curves. As a truly global searching method, we have used the simplexlike algorithm given in the numerical recipes. ${ }^{41}$ However, for reasons explained below, we have also performed a systematic search on a standard grid.

\section{RESULTS AND DISCUSSION}

The surface atomic structure for the $3 \mathrm{D} \mathrm{YSi}_{1.7}$ has been investigated by performing the following steps: discrimination between models, determination of accurate values for the interatomic distances, and refining of the nonstructural parameters involved in the system.

\section{A. Discrimination between models}

In order to clarify the geometrical structure of this silicide, we need to consider different likely atomic configurations. The considered surfaces consist of a bulklike Si bilayer without vacancies in the topmost plane, and with a first neighbor distance of $3.84 \AA$. Y atoms are located on the $\mathrm{H}_{3}$ site with respect to this Si bilayer. In this initial step, we did not take into account neither lateral nor vertical relaxation of the equivalent atoms, and only the interplanar distances of the last four layers (topmost Si bilayer, two yttrium layers and one graphitelike Si layer) were varied. The rest of the planes remain in the ideal bulk position with a distance of $2.07 \AA$ between Si and Y planes. In the inner planes we have considered eight atoms per unit cell in the bulk (three of $\mathrm{Y}$ and five of $\mathrm{Si}$ ) and nine atoms per unit cell at the surface (three $\mathrm{Y}$ atoms and six Si atoms). We have considered different possibilities for the positions of vacancies.

(i) Whether or not there are vacancies in the buckled $\mathrm{Si}$ surface bilayer [Fig. 3(a)] (in the $\mathrm{Si}_{\text {down }}$ layer).

(ii) The position of the vacancies with respect to the topmost $\mathrm{Si}$ bilayer, vacancies stack with the $\mathrm{Si}_{\text {up }}$ or with the $\mathrm{Si}_{\text {down }}$ atoms [Fig. 3(b)].

(iii) The influence of the variation of the stacking of the vacancies in the graphitelike planes, vacancies pile up or rotated $60^{\circ}, 120^{\circ}$, and $180^{\circ}$ in consecutive planes [Fig. 3(c)].

Combining all these possibilities, we have a total of 16 different geometrical models to be minimized. Since LEED is most sensitive to the very surface region, we expect the first point to be more important than the third in the analysis.

From the $R_{P}$ results, we can immediately rule out the presence of $\mathrm{Si}_{\text {down }}$ vacancies in the buckled $\mathrm{Si}$ top bilayer, because usually the $R_{P}$ values for these models were very high, of the order of 0.6. This leads to a surface termination of the silicide with a $\mathrm{YSi}_{2}$ stoichiometry instead of $\mathrm{YSi}_{1.7}$, in good agreement with previous experimental findings. ${ }^{25,42}$

We tried to determine the geometrical arrangement of this buckled Si bilayer with respect to the vacancies position in the last graphitelike Si plane [Fig. 3(b)]. When vacancies are 


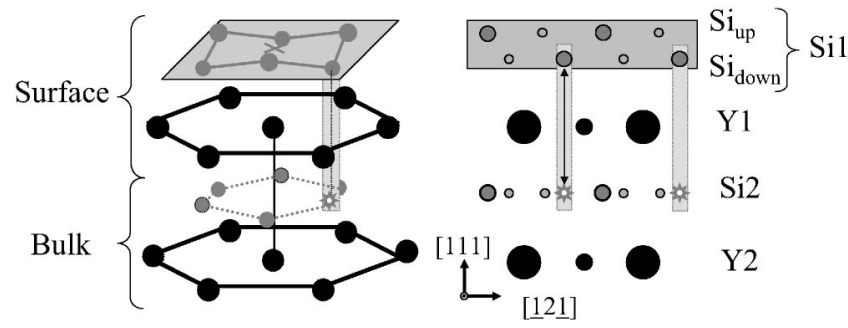

FIG. 4. Schematic representation of the geometrical arrangement found for the $\mathrm{YSi}_{1.7}$ by dynamical LEED-IV calculations.

located under $\mathrm{Si}_{\text {down }}$ atoms, the $R_{P}$ goes down to 0.25 , increasing its value to $R_{P}=0.50$ when vacancies are piled up with the $\mathrm{Si}_{\text {up }}$ atoms.

Testing the stacking of the vacancies in the inner planes we have obtained similar $R_{P}$ values, all of them within the statistical variance RRP. Therefore, we cannot distinguish between the four rotated configurations considered in (iii) for the last two graphitelike $\mathrm{Si}$ planes [see Fig. 3(c)]. LEED provides accurate surface information but has not enough sensibility to distinguish the position of the vacancies in the second graphitelike $\mathrm{Si}$ plane, that it is located approximately $9 \AA$ below the surface. Keeping this in mind, and taking into account the presence of multiple domains, we conclude that the final surface atomic determination will not be affected by the stacking of the vacancies in the inner planes. Therefore, in order to simplify the calculations we have assumed that vacancies are piled up in the bulk of the silicide.

Thus, from the point of view of the LEED analysis, the final surface model consists of a topmost buckled $\mathrm{Si}$ bilayer with no vacancies on top of a Y layer, and with one of the three $\mathrm{Si}_{\text {down }}$ atoms on top of a $\mathrm{Si}$ vacancy (sketched in Fig. 4).

\section{B. Determination of accurate values for the interatomic distances}

We have performed a careful minimization of the $R_{P}$ factor, allowing lateral and small vertical relaxations. As we have mentioned in the experimental and theoretical details section, we have simultaneously performed a search combining an automated search with a manual investigation of the parameters. This process has been performed by the following:

(i) An initial search of the interplanar distance in the surface planes as well as in the bulk planes.

(ii) A subsequent refinement of the $R_{P}$ by allowing every atom to laterally relax its position.

(iii) The final optimization of nonstructural parameters, of which the vibrational amplitude has been the most important. These have been optimized independently for each layer.

These three steps were repeated for every relevant parameter to find a global minimum of the $R_{P}$ that would determine the surface atomic positions.

In the procedure used to adjust the parameters we have considered as the surface region the last $\mathrm{Y}$ layer and the

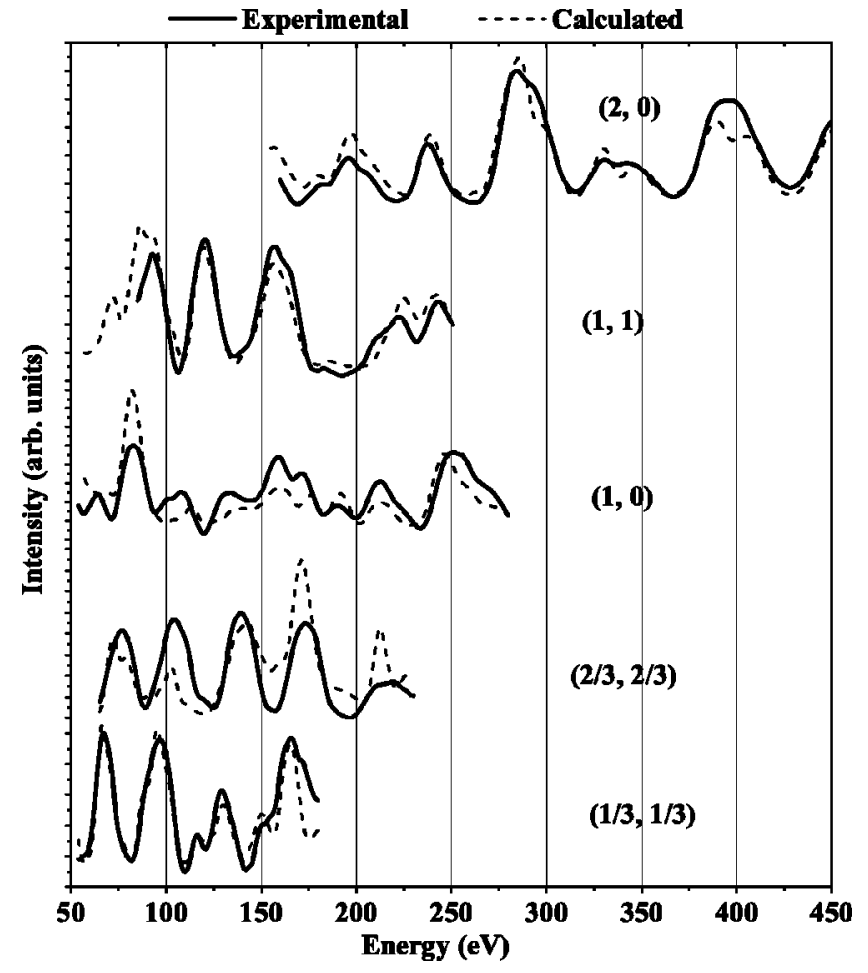

FIG. 5. Experimental and calculated LEED-IV curves for the lowest $R_{P}$.

buckled Si bilayer. In Fig. 4 the schematic distribution is shown. Although the number of atoms involved in the optimization is large, and the optimization process has been quite hard, the final geometry is very convincing as the best $R_{P}$ $(0.20)$ is indeed quite low. In addition, the visual comparison of the experimental $\mathrm{I}(\mathrm{V})$ curves with the theoretical ones (Fig. 5) show a very satisfactory agreement. The structural and nonstructural parameters for this best fit are summarized in Table I, and the final geometry is sketched in Fig. 6, including the lateral and vertical relaxations that we discuss next.

For the last graphitelike Si plane, $\mathrm{Si}$, we have found that the three $\mathrm{Si}$ atoms surrounding the vacancies are laterally displaced $0.36 \pm 0.09 \AA$ from the ideal hexagonal position toward the vacancies. Therefore, there is a deformation of the hexagonal structure, making the $\mathrm{Si}$ bond length to change from $2.21 \AA$ in the stressed ideal plane, to $2.42 \AA$ in the relaxed one. This value is slightly higher (and within the error bar) than the $\mathrm{Si}-\mathrm{Si}$ bond length in a buckled $\mathrm{Si}$ layer, $2.35 \AA$. We notice that this increment makes more uniform all the five $\mathrm{Si}-\mathrm{Si}$ bonds in the $\mathrm{Si}$ planes.

For the Y planes, we have found large error bars for the lateral displacement of the Y atoms $(0.10 \AA$ for the $\mathrm{Y} 1$, and $0.15 \AA$ for the Y2), which are typical of a normal incidence LEED analysis. Previous works related to the bulk structure have shown lateral displacements smaller than our error bars, ${ }^{21}$ that cannot be confirmed from our work given the uncertainty we have on these parameters. Thus we will keep the Y-Y distance as $3.84 \AA$ corresponding to the ideal distance. ${ }^{9}$ In the perfect hexagonal $\mathrm{AlB}_{2}$ structure each $\mathrm{Si}$ atom is surrounded by six $\mathrm{Y}$ atoms at $3.03 \AA$. When the geometry is relaxed, three different distances have been 
TABLE I. In the upper part of this table the interatomic distances between inequivalent atoms calculated by LEED are shown. The Si lateral movement is toward the vacancies. In the lower part the thermal vibrational amplitudes and the Debye-Waller temperatures are summarized.

\begin{tabular}{|c|c|c|c|}
\hline \multicolumn{2}{|c|}{ Vertical distances $(\AA)$} & \multicolumn{2}{|c|}{ Lateral relaxations $(\AA)$} \\
\hline $\mathrm{Y} 1-\mathrm{Si}_{\text {up }}$ & $2.69 \pm 0.06$ & $\mathrm{Si}_{\text {up }}$ & $0.00 \pm 0.40$ \\
\hline $\mathrm{Y} 1-\mathrm{Si}_{\text {down }}$ & $2.05 \pm 0.03$ & $\mathrm{Si}_{\text {down }}$ & $0.00 \pm 0.03$ \\
\hline $\mathrm{Y} 1-\mathrm{Si}_{\text {down }}(\mathrm{vac})$ & $1.97 \pm 0.07$ & $\mathrm{Si}_{\text {down }}(\mathrm{vac})$ & $0.00 \pm 0.03$ \\
\hline Y1-Si2 & $2.07 \pm 0.03$ & $\mathrm{Y} 1$ & $0.00 \pm 0.10$ \\
\hline \multirow[t]{6}{*}{$\mathrm{Si} 2-\mathrm{Y} 2$} & $2.06 \pm 0.03$ & $\mathrm{Si} 2$ (close vac) & $0.36 \pm 0.09$ \\
\hline & & $\mathrm{Si} 2$ (away vac) $^{\mathrm{a}}$ & 0.00 \\
\hline & & Y2 & $0.00 \pm 0.15$ \\
\hline & \multicolumn{3}{|c|}{ Nonstructural parameters } \\
\hline & \multicolumn{3}{|c|}{$\mathrm{V}_{\text {real }}=-10 \mathrm{eV}, \mathrm{V}_{\text {imaginario }}=4 \mathrm{eV}$} \\
\hline & $\langle\Delta r\rangle(\AA)$ & \multicolumn{2}{|c|}{$T_{\text {Debye }}(\mathrm{K})$} \\
\hline $\mathrm{Si}_{\text {up }}$ & 0.22 & \multicolumn{2}{|c|}{219} \\
\hline $\mathrm{Si}_{\text {down }}$ & 0.16 & \multicolumn{2}{|c|}{301} \\
\hline $\mathrm{Y} 1, \mathrm{Y} 2$ & 0.06 & \multicolumn{2}{|c|}{458} \\
\hline $\mathrm{Si} 2$ & 0.08 & \multicolumn{2}{|c|}{594} \\
\hline
\end{tabular}

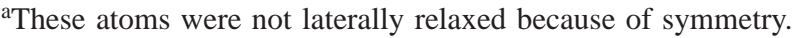

found for the Y-Si bonds. Two short bonds of $2.94 \AA$, two intermediate bonds of $3.01 \AA$ and one of $3.25 \AA$. The distance between the last $\mathrm{Y}$ layer $(\mathrm{Y} 1)$ and the $\mathrm{Si}_{\text {down }}$ plane is nearly equal to the Y-Si bulk layers. This is an indication of a soft interaction between $\mathrm{Si}$ and $\mathrm{Y}$ atoms.

Regarding the topmost $\mathrm{Si}$ atomic positions, we are not sensible to lateral relaxations in the buckled Si bilayer, again because of the large error bar of our statistical analysis associates to this parameter $(0.40 \AA)$. On the other hand, we are more sensitive to vertical displacements and the same analysis allows us to determine a small vertical movement for the $\mathrm{Si}_{\text {down }}$ atom that sits on top of the $\mathrm{Si}$ vacancy. This atom is $0.08 \AA$ lower than their neighbors. The three $\mathrm{Si}_{\text {up }}$ atoms are at the same height, $2.69 \AA$ over the Y1 layer, and do not present vertical distortions, the $\mathrm{Si}_{\text {down }}$ atoms are, in average, at a vertical distance of $2.01 \AA$ over the $\mathrm{Y} 1$, giving a buckling distance of $0.68 \AA$.

We have found that $\mathrm{Si}$ vacancies are located below the $\mathrm{Si}_{\text {down }}$ atoms, indicating that the model with vacancies under the $\mathrm{Si}_{\text {up }}$ (Refs. 31 and 32) is not compatible with our LEED analysis. Our result, however, is in agreement with previous ab initio calculations performed by Magaud et al. ${ }^{22}$ and with STM experiments reported by Martín-Gago et al. ${ }^{33,34}$ In their interpretation of experimental STM images, Martín-Gago et al. proposed a lateral relaxation for the $\mathrm{Si}_{\text {up }}$. Our LEED results do not show such a lateral relaxation. Preliminary results obtained from $a b$ initio calculations to simulate the same STM images indicate that the inequivalent $\mathrm{Si}_{\text {down }}$ contribute to the image inducing a "ghost" lateral relaxation. This effect should then be considered more related to electronic effects than to the true structural disposition of atoms. The work on the theoretical interpretation of STM images is in progress at the present moment in

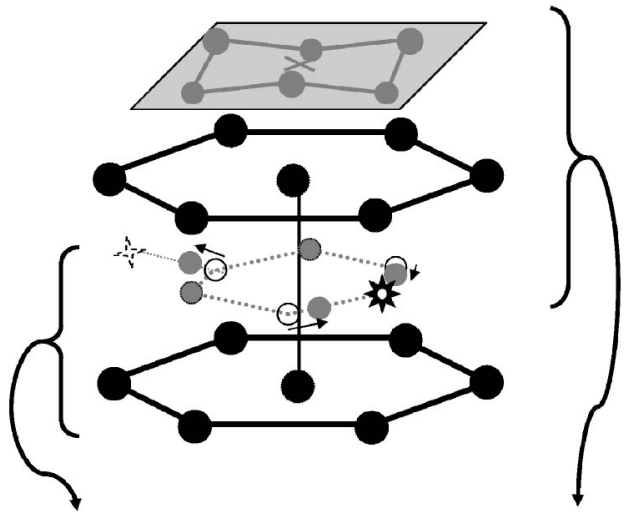

Top view: inner planes

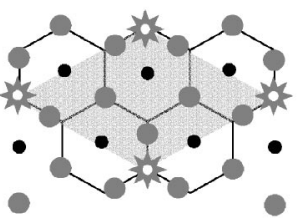

Top view: surface planes

FIG. 6. Schematic representation of the best fit structure of the $\mathrm{YSi}_{1.7}$ where the lateral relaxation is marked.

our group, and it will be reported on a separate paper dealing with the STM problem.

\section{Refining of the nonstructural parameters}

Finally, we find that a correct estimation of the thermal vibrational amplitudes at each plane is necessary to properly minimize the $R_{P}$ factor. To simulate thermal vibrations of atoms we take the standard approach of using a DebyeWaller factor related to a model where atoms vibrate isotropically. We observe that an important drop in the $R_{P}$ can be obtained by optimizing the different root mean squared amplitudes of vibration differently for atoms in the surface region. In particular, if we use $\mathrm{Si}$ bulklike values for the last $\mathrm{Si}$ planes $(0.06 \AA)$ the optimum $R_{P}$ is around 0.42 . This value, on the other hand, drops to 0.20 if we allow for larger vibrational amplitudes $\left(0.22 \AA\right.$, and $0.16 \AA$ for $\mathrm{Si}_{\text {up }}$ and $\mathrm{Si}_{\text {down }}$, respectively). This finding is consistent with our previous investigation for the $2 \mathrm{D}$ phase. ${ }^{12}$ In the lower part of Table I, the best fit values for the nonstructural parameters can be found. Although it might seem natural that atoms near the surface may have larger vibration amplitudes, with the present experimental database we could not separate this effect from static disorder. ${ }^{43}$ To distinguish between both effects it would be necessary to take further experimental data measured at different temperatures.

\section{CONCLUSIONS}

We have determined the atomic positions in the surface of the three-dimensional yttrium silicide epitaxially grown on $\mathrm{Si}(111) 7 \times 7$ by dynamical LEED-IV. We have found that the geometric atomic structure of this silicide surface consists of a hexagonal structure with graphitelike Si planes with an ordered network of vacancies intercalated byY planes. 
The topmost plane is a buckled $\mathrm{Si}$ overlayer with no vacancies. It shows a slightly vertical relaxation of the $\mathrm{Si}_{\text {down }}$ atom sitting over the vacancy position in the last graphitelike $\mathrm{Si}$ plane. The atoms in this bilayer occupy hexagonal positions, whereas the $\mathrm{Si}$ atoms in the interior planes are laterally relaxed in order to homogenize the Si-Si bonds.

\section{ACKNOWLEDGMENTS}

The authors acknowledge helpful advice with previous experiments from Dr. C. Polop and with the CLEED package from Dr. G. Held. This work has been financially supported through Grant No. MAT2002-395.
${ }^{1}$ H. Norde, J. de Sousa Pires, F. M. d'Heurle, F. Pesavento, S. Petersson, and P. Tove, Appl. Phys. Lett. 38, 865 (1981).

${ }^{2}$ K. N. Tu, R. D. Thompson, and B. Y. Tsaur, Appl. Phys. Lett. 38, 626 (1981)

${ }^{3}$ R. D. Thompson, B. Y. Tsaur, and K. N. Tu, Appl. Phys. Lett. 38, 535 (1981).

${ }^{4}$ R. D. Thompson and K. N. Tu, Thin Solid Films 93, 265 (1982).

${ }^{5}$ S. Vandré, C. Preinesberger, and W. Busse M. Dähne, Appl. Surf. Sci. 78, 2012 (2001).

${ }^{6}$ A. Iandelli, A. Palenzona, and G. L. Olcese, J. Less-Common Met. 64, 213 (1979).

${ }^{7}$ R. T. Tung, A. F. L. Levi, and J. M. Gibson, J. Vac. Sci. Technol. B 4, 1435 (1986).

${ }^{8}$ J. A. Knapp and S. T. Picraux, Appl. Phys. Lett. 48, 466 (1986).

${ }^{9}$ R. Baptist, A. Pellissier, and G. Chauvet, Solid State Commun. 68, 555 (1988).

${ }^{10}$ X. Tang, J. Katcki, E. Dubois, N. Reckinger, J. Ratajczak, G. Larrieu, P. Loumaye, O. Nisole, and V. Bayot, Solid-State Electron. 47, 2105 (2003).

${ }^{11}$ P. Wetzel, C. Pirri, G. Gewinner, P. Roge, S. Pelletier, F. Palmino, and J. C. Labrune, Phys. Rev. B 56, 9819 (1997).

${ }^{12}$ C. Rogero, C. Polop, L. Magaud, J. L. Sacedón, P. L. de Andrés, and J. A. Martín-Gago, Phys. Rev. B 66, 235421 (2002).

${ }^{13}$ A. Mharchi, L. Stauffer, S. Saintenoy, C. Pirri, P. Wetzel, D. Bolmont, and G. Gewinner, Solid State Commun. 97, 249 (1996)

${ }^{14}$ F. Arnaud d'Avitaya, A. Pario, J. C. Oberlin, Y. Campidelli, and J. A. Chroboczek, Appl. Phys. Lett. 54, 2198 (1989).

${ }^{15}$ R. Baptist, S. Ferrer, G. Grenet, and H. C. Poon, Phys. Rev. Lett. 64, 311 (1990).

${ }^{16}$ N. Frangis, G. Van Tendeloo, J. Van Landuyt, P. Muret, and T. A. Nguyen Tan, Appl. Surf. Sci. 102, 163 (1996).

${ }^{17}$ J. Y. Veuillen, S. Kennou, and T. A. Nguyen Tan, Solid State Commun. 79, 795 (1991).

${ }^{18}$ C. Wigren, J. N. Anderson, R. Nyholm, and K. O. Karlson, J. Vac. Sci. Technol. A 9, 1942 (1991).

${ }^{19}$ W. A. Henle, H. G. Ramsey, F. P. Netzer, R. Cimino, and W. Braun, Solid State Commun. 71, 657 (1989).

${ }^{20}$ L. Magaud, J. Y. Veuillen, D. Lollman, T. A. Nguyen Tan, D. A. Papaconstantopoulos, and M. J. Mehl, Phys. Rev. B 46, 1299 (1992).

${ }^{21}$ M. Lohmeier, W. J. Huisman, E. Vlieg, A. Nishiyama, C. L. Nicklin, and T. S. Turner, Surf. Sci. 345, 247 (1996).

${ }^{22}$ L. Magaud, A. Pasturel, G. Kresse, and J. Hafner, Phys. Rev. B 55, 13479 (1997).
${ }^{23}$ L. Stauffer, C. Pirri, P. Wetzel, A. Mharchi, P. Paki, D. Bolmont, and G. Gewinner, Phys. Rev. B 46, 13201 (1992).

${ }^{24}$ L. Stauffer, A. Mharchi, S. Saintenoy, C. Pirri, P. Wetzel, D. Bolmont, G. Gewinner, and C. Minot, J. Phys. Chem. Solids 58, 567 (1997).

${ }^{25}$ L. Stauffer, A. Mharchi, S. Saintenoy, C. Pirri, P. Wetzel, D. Bolmont, and G. Gewinner, Phys. Rev. B 52, 11932 (1995).

${ }^{26}$ G. H. Shen, J. C. Chen, and L. J. Chen, Appl. Surf. Sci. 142, 300 (1999).

${ }^{27}$ A. Noya, M. B. Takeyama, K. Sasaki, E. Aoyagi, and K. Hiraga, J. Appl. Phys. 85, 2934 (1999).

${ }^{28}$ M. Lohmeier, W. J. Huisman, G. ter Horst, P. M. Zanwijn, A. Nishiyama, C. L. Nicklin, T. S. Turner, and E. Vlieg, Proceedings fall meeting, 1994.

${ }^{29}$ R. Gunnella, J. Y. Veuillen, T. A. Nguyen Tan, and A. M. Flank, Phys. Rev. B 57, 4154 (1998).

${ }^{30}$ P. Paki, U. Kafader, P. Wetzel, C. Pirri, J. C. Peruchetti, D. Bolmont, and G. Gewinner, Phys. Rev. B 45, 8490 (1992).

${ }^{31}$ T. P. Roge, F. Palmino, C. Savall, J. C. Labrune, P. Wetzel, C. Pirri, and G. Gewinner, Phys. Rev. B 51, 10998 (1995).

${ }^{32}$ T. P. Roge, F. Palmino, C. Savall, J. C. Labrune, and C. Pirri, Surf. Sci. 383, 350 (1997).

${ }^{33}$ J. A. Martín-Gago, J. M. Gómez-Rodríguez, and J. Y. Veuillen, Surf. Sci. 366, 491 (1996).

${ }^{34}$ J. A. Martín-Gago, J. M. Gómez-Rodríguez, and J. Y. Veuillen, Phys. Rev. B 55, 5136 (1997).

${ }^{35}$ J. B. Pendry, Low-Energy-Electron Diffraction (Academic, London, 1974).

${ }^{36}$ C. Rogero, C. Polop, J. L. Sacedón, and J. A. Martín-Gago, Surf. Interface Anal. 36, 1195 (2004).

${ }^{37}$ C. Polop, C. Rogero, J. L. Sacedón, and J. A. Martín-Gago, Surf. Sci. 482, 1337 (2001).

${ }^{38}$ G. Held, gh10009@hermes.cam.ac.uk, Universidad de Cambridge, U.K.

${ }^{39}$ M. A. Van Hove, W. H. Weinberg, and C. M. Chan, Low-Energy Electron Diffraction (Springer-Verlag, Berlin, 1986).

${ }^{40}$ J. Pendry, J. Phys. C 13, 937 (1980).

${ }^{41}$ W. H. Press, S. A. Teukolsky, W. T. Vetterling, and B. P. Flannery, Numerical Recipes (Cambridge University Press, Cambridge, 1992).

${ }^{42}$ P. Wetzel, S. Saintenoy, C. Pirri, D. Bolmont, and G. Gewinner, Phys. Rev. B 50, 10886 (1994).

${ }^{43}$ V. Blum, L. Hammer, W. Meier, and K. Heinz, Surf. Sci. 488, 219 (2001) 